International Journal of Pure and Applied Mathematics

Volume 110 No. 3 2016, 563-569

ISSN: 1311-8080 (printed version); ISSN: 1314-3395 (on-line version)

url: http://www.ijpam.eu

doi: 10.12732/ijpam.v110i3.14

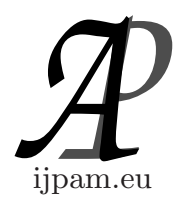

\title{
RESEARCH AND SIMULATION OF IMAGES \\ CLASSIFICATION ALGORITHM FOR DISTRIBUTED \\ OBJECTS OBTAINED BY REMOTE SENSING OF \\ THE EARTH'S SURFACE
}

\author{
Leonid Dorosinskiy ${ }^{1}$, Andrey Sosnovsky $^{2}$, Nina Vinogradova ${ }^{3}$ \\ ${ }^{1,2,3}$ Ural Federal University \\ 620002, Mir Street 19, Ekaterinburg, RUSSIAN FEDERATION
}

\begin{abstract}
Application of ultra-wideband signals and large apertures makes it possible to obtain a sufficiently detailed radar image of a spatially distributed object at the processing unit output. But the problem of optimal classification of synthetic aperture radar (SAR) data remains relevant due to specific features of radar images. The paper describes solution of the synthesis problem for optimal SAR image classification algorithm. The optimal set of very informative and small dimension features is found and features based on the moments of SAR images are proposed. A comparative analysis of classification algorithms using various features is made, and the ratio is proposed, which can be used as the classification sign that is invariant to object shift and to specific distortions caused by the object rotation.
\end{abstract}

AMS Subject Classification: 54Cxx, 93A30, 94A12

Key Words: image classification algorithm, synthetic aperture radar, remote sensing

\section{Introduction}

Application of ultra-wideband signals and large apertures makes it possible to obtain a sufficiently detailed radar image of the spatially distributed object at the processing unit output [1]-[4]. One of the main tasks for developers of such

Received: $\quad$ August 27, 2016

Revised: $\quad$ October 6, 2016

Published: November 5, 2016 (c) 2016 Academic Publications, Ltd. url: www.acadpubl.eu 
images processing unit is development of the efficient classification algorithms of radar image in the presence of distortions. Such distortions are simulated by limited resolving power of receiving aperture, fluctuations of observable signal, and interferences. At the same time, it is essential to choose a feature vector combining high information content with its rather small size. Such a vector can be built on the basis of sufficient statistics or radar image moments [1]. The second part of the mentioned problem includes finding the efficient and simple solution rules.

\section{Classification Algorithm}

The field made by reflected signal in the aperture of receiving antenna can be presented in the next form:

$$
\dot{U}_{A}(t, r)=\int_{Q_{k}} \dot{\alpha}(t, r, q) \sigma_{k}(q) f(t, r, q) d q+\dot{n}(t, r),
$$

where $r$ and $q$ are position vector of the receiving aperture and position vector of the observable object correspondingly; $\dot{\alpha}(t, r, q)$ is the weight function depending on characteristics of receiving aperture and geometric ratio that associate object coordinate and aperture, $\sigma_{k}^{2}(q)$ is the source image of the $k$-class object, i.e. the distribution of signal power reflected by object according to its coordinates within the $Q_{k}$ spatial region; $f(t, r, q)$ is the random fluctuation field determining multiplicative distortions $\sigma_{k}^{2}(q)$ of the information field; $\dot{n}(t, r)$ is the Gaussian field of additive interference.

If the number of resolution elements on the object surface is rather large, the observable field (1) is considered to be Gaussian. In this case, the statistics corresponded with the $k$-class observation can be written in the next form:

$$
\lambda_{k}=\iint_{T} \iint_{L} U_{A}\left(t_{1}, r_{1}\right) U_{A}\left(t_{2}, r_{2}\right) W_{k}\left(t_{1}, t_{2}, r_{1}, r_{2}\right) d t_{1} d t_{2} d r_{1} d r_{2},
$$

where $T$ and $L$ are the observation time and spatial region taken by antenna system respectively; $W_{k}\left(t_{1}, t_{2}, r_{1}, r_{2}\right)$ is the weight function of processing corresponded with the $k$-class of recognizable ones.

Equation (2) implies that mathematical mean of field (1) is equal to zero. This limitation is not essential because the information parameter of considered task has the specific density of fluctuation of the average power $\sigma_{k}^{2}(q)$. Furthermore, two assumptions are introduced, that are usually hold in practice. Observation time and sizes of antenna system considerably exceed correlation time 
and interval of spatial correlation of the received signal. Signals from separate elements of spatially distributed object are statistically independent.

At the same time, the weight function of processing can be calculated from the integral inversion equation by the Fourier method [1] and equation for the $k$-component of sufficient statistic vector will have the next form:

$$
\lambda_{k}=\int_{Q_{k}} \frac{\sigma_{k}^{2}(q)}{1+\sigma_{k}^{2}(q)} z(q) d q
$$

where

$$
z(q)=\left|\int_{T} \int_{L} U_{A}(t, r) \dot{\alpha}(t, r, q) d t d r\right|^{2}
$$

is spatially distributed object on the radar image.

The next method of feature vector formation is based on calculation of the moments various degrees of $z(q)$ function [2]; and the central moment $p_{1}+p_{2}+$ $\ldots+p_{n}$ is determined by the formula:

$$
\mu_{p_{1}, p_{2}, \ldots, p_{n}}=\int_{Q}\left(q_{1}-q_{10}\right)^{p_{1}}\left(q_{2}-q_{20}\right)^{p_{2}} \ldots\left(q_{n}-q_{n 0}\right)^{p_{n}} z(q) d q
$$

where $q_{i 0}=\int_{Q} q_{i} z(q) d q / \int_{Q} z(q) d q$.

The article compares several parametric and non-parametric methods of solution. The first method assumes that the attribute vector has multidimensional regular distribution with the mathematical means and covariance matrices estimated on the grade level. The first solution is found with the minimum function: $\tilde{i}=\min _{i}\left\{\left(\Delta-\tilde{M}_{i}\right)^{t} \tilde{\sum}_{i}^{-1}\left(\Delta-\tilde{M}_{i}\right)\right\}+\ln \left(\tilde{\sum}_{i}\right)$, where $\tilde{i}$ is the number estimation of object class; $\Delta=\left\{\lambda_{1}, \lambda_{2}, \ldots, \lambda_{n}\right\}$ is the attribute vector; $\tilde{M}_{i}$ is the estimation vector of the average means for the $i$-class objects; $\tilde{\sum}_{i}$ is the covariance matrix estimation of the attribute vector for the $i$-class.

The second solution was received by simplification based on assumption of separate components independence of attribute vector. Moreover, there are shown the results of comparison with the nonparametric rule $-K$-nearest neighbors method [1] (method 3).

The basic statistical data used for algorithm investigation are received by modeling the $2 \mathrm{D}$ radar images that are relevant to signal field at the processing unit output in the side-looking station with synthetic aperture [2]. The observable object is simulated with separate blind targets and diffusive component. 
Recognizable classes differ in location of glitter points. There are 3 classes. Vectors are used as signs: sufficient statistics $\lambda=\left\{\lambda_{1}, \lambda_{2}, \lambda_{3}\right\}$, and moments $\mu_{1}=\left\{\mu_{20}, \mu_{00}, \mu_{02}\right\}$ and $\mu_{2}=\left\{\mu_{30}, \mu_{20}, \mu_{00}, \mu_{02}, \mu_{03}\right\}$. Probability of correct detection according to three described solution rules for each vector are distributed in the way, shown in Table 1.

\begin{tabular}{|r|r|r|r|}
\hline \multirow{2}{*}{ Sign } & \multicolumn{3}{|c|}{ Solution rule } \\
\cline { 2 - 4 } & Method 1 & Method 2 & Method 3 \\
\hline$\lambda_{1}$ & 0.98 & 0.80 & 0.94 \\
\hline$\mu_{1}$ & 0.76 & 0.58 & 0.65 \\
\hline$\mu_{2}$ & 0.60 & 0.50 & 0.54 \\
\hline
\end{tabular}

Table 1: Solution rules

The received results show under detection of a small number of classes (typical situation for radar image) that sufficient statistic vector provides higher probability of correct classification than moment vector of the same or a little bigger size. Solution supposing the statistical independence of signs (method 2) appreciably gives poor result against rules, which take into account their dependence (methods 1 and 3).

Sufficient statistic vector considerably depends on supposed distribution of specific backscatter over observable target surface. The usage of this vector does not allow one to compute some essential features. This happens under forming the real images, which are taken from objects with unknown parameters of their linear movement and rotation w.r.t. the gravity center. This paper proposes signs invariant to rotation of observable object and to its center shift. The proposed signs are based on the images moments.

The radial component of object point the motion speed $\left(V_{r}\right)$ leads to the shift of its image, formed in the SAR by the size proportional to this radial speed. At the same time, form of the processing system response to the reflected signal from such point (impulsive system reaction) remains invariable.

It holds $V_{r}=x \omega$ for points of space-distributed object in rotation about the center, where $x$ is the azimuthal point data counted from the center of object rotation, $\omega$ is the angular rotation speed. In connection with it, the image shift of the target points will be proportional to $x$-coordinate value. In the whole, the object image will extend and contract in azimuth depending on rotation speed direction.

The equation for representation of moving object in discrete form is shown 
in [2]. For the continuous case, we can get the similar dependence

$$
\phi(x, y)=\int A_{k}(\xi, y) D(x-\beta \xi) d \xi+n(x, y)
$$

where $A_{k}(x, y)$ is the distribution of complex coefficient reflection of the $k$-class object of $X$ and $Y$ axes; $D(x)$ is the impulsive reaction of processing unit with $\operatorname{sinc}(x)$-form; $\beta=1+2 \omega$ is the coefficient considering the object rotation; $n(x, y)$ is the Gaussian noise.

There is the problem to find the link between the moments of distorted image (6) and undistorted one considering only signal component in (6). The equation of distorted image is received by substitution of $\beta=1(\omega=0)$ values into (6)

$$
\Phi_{0}(x, y)=\int A_{k}(\xi, y) D(x-\xi) d \xi
$$

It is known that moments are coefficients of the Taylor series expansion of 2D Fourier spectrum image, i.e.

$$
S(u, \nu)=\sum_{p=0}^{\infty} \sum_{q=0}^{\infty} m_{p q} \frac{(i u)^{p}}{p !} \frac{(i u)^{q}}{q !}
$$

where

$$
S(u, \nu)=\iint \Phi(x, y) \exp [-i(u x+\nu y)] d x d y
$$

is the image spectrum;

$$
m_{p q}=\iint x^{p} y^{q} \Phi(x, y) d x d y
$$

is the image moment of $p+q$ degree. It is obvious that

$$
m_{p q}=(-1)^{p+q} \frac{\partial^{(p+q)}}{(\partial u)^{p}(\partial \nu)^{q}}[S(u, \nu)]_{\nu=u=0},
$$

and equation for spectrum of undistorted image (7) can be written in the next form:

$$
S_{0}(u, \nu)=\iint \Phi_{0}(x, y) \exp [-i(u x+\nu y)] d x d y=S_{A}(u, \nu) S_{D}(u),
$$

where $S_{A}(u, \nu)=\iint A_{k}(x, y) \exp [-i(u x+\nu y)] d x d y$, 
$S_{D}=\int D(x) \exp [-i u x] d x$. Similarly to distorted image $(\beta \neq 1)$, we get

$$
S_{0}(u, \nu)=S_{A}(\beta u, \nu) S_{D}(u)
$$

since $D(x)$ function has $\operatorname{sinc}(x)$ form and its spectrum $S_{D}(u)$ is right-angled. Therefore, calculating partial derivative in (11), we can consider $S_{D}(u)=$ const.

Using change of variables $\phi=\beta u$ and evident equality $(\partial u)^{p}=\left(\frac{1}{\beta}\right)^{p}(\partial \phi)^{p}$, we get the identity

$$
\frac{\partial^{(p+q)}}{(\partial u)^{p}(\partial \nu)^{q}}[S(u, \nu)]_{u=\nu=0}=\beta^{p} \frac{\partial^{(p+q)}}{(\partial \phi)^{p}(\partial \nu)^{q}}\left[S_{0}(\phi, \nu)\right]_{\phi=\nu=0}
$$

From (14) and (11) follows

$$
m_{p q}=b^{p} m_{p q}^{0}
$$

where $m_{p q}^{0}=\iint x^{p} y^{q} \Phi_{0}(x, y) d x d y$ is the moment of undistorted image of $p+q$ degree. With $p=1$ from equation (15), we get $\beta=\left(\frac{m_{1 q}}{m_{1 q}^{0}}\right)$. At the same time, equation (15) turns into the form

$$
m_{p q}=\left(\frac{m_{1 q}}{m_{1 q}^{0}}\right)^{p} m_{1 q}^{0}
$$

and the identity follows

$$
\frac{m_{p q}}{\left(m_{1 q}\right)^{p}}=\frac{m_{p q}^{0}}{\left(m_{1 q}^{0}\right)^{p}}
$$

which confirms $m_{p q} /\left(m_{1 q}\right)^{p}$ independence on the speed rotation of the observable object. Thereby, signs $I_{p q}=m_{p q} /\left(m_{1 q}\right)^{p}$ can be used as invariants to specific radar image distortion received by the SAR and caused by rotation of the observable object. It should be noted that moments (10) have essential fault that complicates their usage. Namely, the moments are not invariant to the shift object gravity center. Calculation central moments over the radar image is more preferable [3]

$$
\mu_{p q}=\iint\left(x-x_{0}^{p}\left(y-y_{0}\right)^{q} \Phi(x, y) d x d y\right.
$$

where $x_{0}=m_{10} / m_{00}$ and $y_{0}=m_{01} / m_{00}$ are coordinates of the radar image gravity center. Using the known ratio

$$
(a-b)^{n}=\sum_{k=0}^{n} C_{n}^{k}(-b)^{k}(a)^{n-k}
$$


we can get the link between moments (10) and (18)

$$
\mu_{p q}=\sum_{n=0}^{p} \sum_{k=0}^{q} C_{p}^{n} C_{q}^{k}(-1)^{n+k} \frac{\left(m_{10}\right)^{n}\left(m_{01}\right)^{k}}{\left(m_{00}\right)^{n+k}} m_{p-n, q-k} .
$$

Expressing all the moments of the right part by the formula (15), we get $\mu_{p q}=\beta^{p} \mu_{p q}^{0}$, where $\mu_{p q}^{0}=\iint\left(x-x_{0}^{0}\right)^{p}\left(y-y_{0}\right)_{0}^{0} \Phi(x, y) d x d y$ are the central moments of the distorted image, $x_{0}^{0}=m_{10}^{0} / m_{00}^{0}$ and $y_{0}^{0}=m_{01}^{0} / m_{00}^{0}$ are normalized coordinates of the gravity center.

\section{Conclusion}

Formula (20) is entirely identical to formula (15). Hence, the ratio $M_{p q}=$ $M_{p q} /\left(\mu_{1 q}\right)^{p}$ can be used as the classification sign that is invariant to each of the object shift w.r.t. the radar image center and to specific distortions caused by the object rotation.

\section{References}

[1] L.G. Dorosinsky, A synthesis of an algorithm for the detection of spatially distributed objects by side-looking radar data, In: Radio-Technical Systems of Space Distribution Facilities Location. Optimization and Modeling, Publishing house UPI Publishing house, Sverdlovsk (1981), 48-52.

[2] L.G. Dorosinsky, T.M. Shitova, Statistical modeling of two-dimensional image spatiallydistributed object, In: Problems of Radio Systems Efficiency and Quality Increasing, UPI Publishing house, Sverdlovsk (1980), 99-101.

[3] M.K. Hu, Shape recognition by invariant relations between the moments, TIIER, 9 (1961).

[4] O.Yu. Ivanov, A.S. Vorobyova, Research of neural network algorithm of classification of remote sensing data, In: 24-rd International Crimean Conference "Microwave and Telecommunication Technology”, CriMiCo'2014, Sevastopol (2014), 1215-1216. 
